\title{
Los sistemas de conexiones entre los espacios web de las agencias de noticias informativas
}

\author{
Cristina Faba Pérez \\ María Victoria Nuño Moral \\ Universidad de Extremadura (España)
}

\section{Resumen}

El presente trabajo se estructura en dos partes. Por un lado, se analiza cuáles son la organización y los servicios informativos disponibles en las principales agencias de noticias tanto nacionales como internacionales. Por otro, el estudio se centra en comparar el comportamiento de los espacios web de las agencias de noticias informativas (ANI), atendiendo a las co-sitas que se generan entre ellos, e incorporando el PageRank de Google como indicador comparativo. El análisis de citas en las publicaciones científicas es un proceso complejo que estudia la relación entre las citas/referencias y las partes que participan en él, el que cita y el citado. Una de las técnicas más consolidadas en este campo es el análisis de cocitas, que estudia las relaciones y frecuencias de las parejas de documentos que son citados conjuntamente por otros documentos posteriores a ellos. El objetivo es descubrir la estructura intelectual de las disciplinas partiendo de la premisa de que cuanto mayor sea el número de veces que dos documentos aparezcan citados conjuntamente mayor es la posibilidad de que su contenido esté relacionado. Las agencias internacionales que presentan una relación más estrecha son Reuters y Associated Press; y las nacionales, EFE y Europa Press. El número de co-sitas correlaciona positivamente con la antigüedad de la agencia en el caso de las nacionales y, en todos los casos, como era previsible, con el PageRank de Google.

Palabras clave: Agencias de noticias informativas (ANI). Espacios web. Webmetría. Análisis de co-sitas.

\section{Abstract}

The present dissertation is structured in two parts. On the one hand, it analyzes what organization and informative services are available in the principal news offices, both national and international. On the other hand, the work compares the behaviour of the web spaces of the agencies of the informative news (AIN), from the cositations that are generated within that environment, and adding the PageRank (Google) as comparative indicator. The citation analysis of scientific publications

Scire. 11 : 2 (sep.-dic. 2005) 141-152. ISSN 1135-3761. 
is a complex process that studies the relationship between the citation/references and the participating elements, citors/citees. One of the most consolidated techniques in this field is cocitation analysis. The objective is simply to discover the intellectual structure of the given discipline, starting from the premise that the more often two documents appear cited conjointly, the more likely it is that their content is related. The international agencies that show a closer relation are Reuters and Associated Press; for the national ones, they are EFE and Europa Press. The number of co-sites correlates positively with the age of the agency in the case of the national ones; and, in all the cases and not surprisingly, with Google PageRank.

Keywords: Agencies of the informative news (AIN). Web spaces. Webmetrics. Cositation analysis.

\section{Los espacios web de las agencias de noticias informativas (ANI)}

El presente trabajo hace referencia principalmente a dos aspectos. Por un lado, en este apartado se analiza la planificación adoptada y los principales servicios con los que cuenta el usuario cuando accede a los diferentes espacios web de las agencias de noticias descritas en el estudio (Reuters, Associated Press, France Press, EFE, Europa Press y Colpisa); por otro, en el apartado 2 se estudian los sistemas de conexiones establecidos entre dichos espacios web.

Usamos la expresión espacio web porque, aunque en castellano podemos diferenciar entre sitio, sede y página web (Aguillo, 1998), en inglés no existe voz para el término sede web por lo que la expresión espacio web, empleada originalmente por Smith (1999), se puede usar para referirnos a tres tipologías diferentes de URL: sitios web —incluso personales_ y conjuntos de páginas web alojadas en otros servidores.

La incursión de las agencias de noticias en la Red ha modificado, en cierta medida, el concepto tradicional que se tenía de ellas. Tradicionalmente, se consideraba a estas agencias como aquellas "empresas dedicadas a la búsqueda, obtención y elaboración de productos informativos, escritos o gráficos, para su posterior distribución entre sus abonados a cambio de una contraprestación económica" (Manfredi, 2000).

En los últimos años, se ha asistido a un cambio en esta concepción desde diferentes perspectivas: por un lado, cualquier usuario podrá tener acceso a la información de última hora ofertada gratuitamente por parte de estas empresas; por otro, el número de servicios, así como el material informativo y documental, se ha incrementado considerablemente (dosier multimedia, infografías, hemerotecas, fototecas, videotecas o fonotecas en línea, etcétera).

De tal forma que podemos afirmar que las agencias de noticias ya no son solo las principales fuentes de información de las que se nutren los diferentes medios 
de comunicación, sino que con la llegada de Internet se han convertido en medios informativos por sí mismas.

\subsection{Agencias internacionales}

La primera agencia internacional creada en el mundo, en 1835, fue France Press (http://www.afp.com) — su origen supone una "continuación" de la agencia francesa Havas-, siendo además pionera en instalarse en América Latina. En la actualidad lidera la información en Asia, siendo la primera agencia en lengua árabe y la de mayor presencia en África.

Como ocurre con los otros dos casos analizados en este apartado, carece de una distribución u organización por secciones. Presenta las noticias de actualidad junto con las fotografías más relevantes, ya que son las dos opciones con las que cuenta el usuario para acotar la búsqueda ("actualidad en imágenes" y "actualidad en texto".

Uno de los recursos que encontramos en los casos analizados es la denominada galería de imágenes, donde se presentan las fotografías acompañadas del pie de foto o texto explicativo de las mismas. De los servicios que destaca en el margen izquierdo, cabría resaltar fundamentalmente dos: "AFP a su medida", donde el usuario recibe diariamente por e-mail la información, y "AFP-directo", que consiste en un sistema de alerta mediante palabras clave sobre diferentes aspectos de la actualidad que pueden clasificarse de forma general como información general, deportes y canales especializados temáticamente, sobre todo dedicados al sector económico.

La agencia americana Associated Press (http://www.ap.org) nace en 1848 y, a diferencia del caso anterior, presenta noticias y fotografías, así como gráficos, vídeos y documentos de audio, llegando a superar el billón de informaciones suministradas diariamente.

En la primera página, junto con los servicios presenta las noticias más destacadas, de tal forma que el acceso a ellas se hace directamente a través del titular correspondiente o mediante el cuadro de búsqueda que se muestra en la parte inferior derecha. Cuando se selecciona una noticia se ofrece el texto de la misma junto con todos los materiales relacionados con dicha información: fotografías, vídeos, dosieres interactivos, otras noticias relacionadas, etcétera.

En cuanto a las búsquedas, cuenta con una búsqueda simple donde el usuario puede determinar, primero, si esa búsqueda se realiza por relevancia o por los documentos más recientes y, en segundo lugar, el período cronológico en el que se tiene que acotar la consulta (en los últimos dos años o desde 1997, fecha origen de los documentos almacenados).

Por otro lado, también presenta una búsqueda avanzada donde, junto a las opciones anteriores, se puede establecer un rango de fechas determinado, así como Scire. 11 : 2 (sep.-dic. 2005) 141-152. ISSN 1135-3761. 
indicar el nombre del autor, o bien los términos relevantes del titular de la información.

Por último, la agencia británica Reuters (http://www.reuters.com), que nace en 1851, está reconocida como la principal agencia de noticias multimedia del mundo (televisión, vídeo, titulares, fotografías, gráficos y servicios on-line). Entre sus usuarios destacan no solo los medios de comunicación, sino también los mercados corporativos y los servicios financieros, que suponen el $90 \%$ de sus ingresos. En la portada presenta las últimas noticias de actualidad destacando las dos o tres más relevantes acompañadas del lead y fotografía correspondiente. El resto se muestra en titulares junto con el icono correspondiente que señala al usuario la existencia de material fotográfico relacionado con dicha información.

Desde esa primera página o portada, el usuario tiene acceso a la galería de imágenes, que se organiza en dos bloques: por un lado, la selección de noticias de las últimas veinticuatro horas; por otro, dosieres fotográficos sobre noticias de información general que no siempre están directamente relacionadas con la actualidad. Cuando el usuario elige el material que quiere visualizar se muestra el conjunto de imágenes relacionadas con el tema seleccionado, cada una de ellas acompañada de su texto explicativo.

Hemos indicado que se trata de la agencia de noticias multimedia más grande del mundo; a diferencia de otros casos mostrados, permite el acceso al canal denominado Reuters $T V$, en el cual el usuario accede a la información de actualidad en movimiento. Presenta una distribución de canales temáticos o por secciones.

Respecto a las búsquedas, estas se realizan por términos — en el caso tanto de información escrita como fotográfica- mediante un cuadro de texto, mostrando en la pantalla el número de documentos, el titular, la fecha y el correspondiente pie de foto, cuya función es la descripción y contextualización de la imagen en relación con el acontecimiento.

\subsection{Agencias nacionales}

De las agencias nacionales, EFE (http://www.efe.es) es, sin duda, la más relevante del panorama español. Fundada en 1939, su origen se remonta al Centro de Corresponsales creado en 1865, considerado como la primera agencia de noticias de España. En su portada, junto al acceso a los diferentes servicios de televisión, radio o reportajes, el usuario encuentra la distribución de noticias por secciones: España, Deportes, Economía, Cultura, etcétera. Muestra las noticias más importantes, destacando aquella de mayor relevancia informativa.

La búsqueda de las noticias se puede realizar de dos formas: una sencilla, mediante un cuadro de texto - por palabras separadas mediante coma-, por sección, o bien utilizando operadores que condicionen la consulta (operadores booleanos y/o frase exacta). Dicha consulta se puede realizar respecto a los últimos quince días. 
En el caso de las fotografías, cuenta con una fototeca considerada el mayor banco de imágenes dentro del mundo de habla hispana, con acceso a más de un millón de imágenes tanto nacionales como internacionales. Junto a este servicio encontramos otros tres relacionados, tales como "fotografías del día" (principales acontecimientos de la jornada), "reportajes" o "infografías". Dentro de este servicio de fototeca, el usuario cuenta con dos tipos de búsquedas. La primera, búsqueda simple, permite indicar el nivel de relevancia o actualidad informativa, la sección y la subsección, y el rango de fechas y/o nombre del personaje que quiere visualizar en imagen. La búsqueda avanzada cuenta, además, con la posibilidad de señalar el país, la ciudad y la responsabilidad tanto física como jurídica de la imagen.

La agencia privada Europa Press (http://www.europapress.es/europa2003), fundada en España en 1957, muestra una planificación similar a la adoptada por la agencia EFE, con área de noticias, reportajes, televisión, etcétera. En este sentido, cabría mencionar que ambas agencias cuentan con un área de Internet donde ofrecen tanto contenidos gratuitos como de pago. Al igual que en el caso anterior, se observa una organización por secciones, más o menos afín a otros medios de comunicación. Presenta las noticias más destacadas de la actualidad acompañadas de su fotografía correspondiente, que pasará a engrosar la galería de imágenes.

Dada su similitud con el caso anterior, simplemente cabría destacar el área de infografías y vídeo streaming, la cual se presenta a modo de dosieres temáticos interactivos, reportajes que no siempre están relacionados con la más inmediata actualidad.

En último lugar, se hace referencia a la agencia de noticias más joven, Colpisa (http://www.colpisa.com), que aparece en España en 1972 por iniciativa de un grupo de diarios regionales y que, a pesar de las diferencias respecto a los ejemplos anteriores, colabora de forma destacable en prensa escrita.

Su web se centra en información escrita, y en su primera página presenta al usuario tres opciones: noticias de última hora, información general e información económica. El servicio de última hora cuenta con una doble posibilidad: por un lado presenta, en un ticker, las últimas diez noticias más destacadas a modo de teletipo; por otro, cuenta con un "monitor de últimas noticias", donde aporta al usuario la información general de todas las secciones o de aquella que elija en cada momento. Cabe destacar que dentro de cada sección se han establecido una serie de "temas" a modo de subcategorías. Ambas opciones de última hora pueden ser personalizadas por el usuario en su propia pantalla de ordenador.

De los otros dos servicios restantes, nos centramos en el de Información General. Aporta al usuario las noticias relevantes haciendo mención tanto de la sección como de la existencia de otras informaciones relacionadas con ese mismo tema. De tal forma que el usuario encuentra dos iconos: "principal" y "tema"; este último indica al lector que existen más noticias relacionadas.

Scire. $11: 2$ (sep.-dic. 2005) 141-152. ISSN 1135-3761. 
A su vez, dada la rapidez que caracteriza al trabajo periodístico, puede ocurrir que, como el servicio de información es permanente, haya noticias que contengan datos erróneos y estos sean conocidos y corregidos posteriormente.

Por último, en relación con las búsquedas, permite la consulta gratuita de informaciones tanto de la jornada en curso como dentro de los últimos treinta días; permite al usuario acotar la consulta por secciones e indicar si los términos de búsqueda han de estar en toda la noticia o exclusivamente en el titular.

\section{Los sistemas de conexiones entre los espacios web de las agencias de noticias informativas (ANI)}

\subsection{Introducción}

Una vez analizada la estructura de los espacios web de las ANI (apartado 1), vamos a descubrir algunas de las relaciones que se establecen entre ellos, no sin antes realizar una pequeña introducción sobre la medición de información en Internet, que nos permitirá enmarcar esta parte del estudio.

En este sentido, el ritmo actual de incremento de los recursos electrónicos disponibles en Internet ha hecho que el usuario final de dicha información se haya visto sobrepasado por la gran cantidad y diversidad de los contenidos, su alta volatilidad o las dudas acerca de la calidad última de ciertos documentos. Para paliar estos problemas, ha sido imprescindible la aplicación y el desarrollo de indicadores que evalúen diferentes rasgos de Internet. Por ello, desde mediados de los noventa, se están investigando y desarrollando indicadores cibermétricos y webmétricos que miden, respectivamente, distintos aspectos de Internet y de la Red de forma cuantitativa.

Básicamente, estos indicadores se dividen en dos conjuntos: el primero se centra en el análisis de las características de la información electrónica disponible en la Red (Smith, 1997; Clausen, 1999; Codina; 2000; Jiménez-Piano, 2001; Novljan y Zumer, 2004...) y el segundo lo forman aquellos indicadores basados en el entramado de enlaces que se establecen entre los espacios web. En este último grupo nos centramos en el presente trabajo.

Los indicadores basados en enlaces sirven para entender el comportamiento social de la Red (Thelwall, 2001; Thelwall y Smith, 2002...) o para llevar a cabo investigaciones relacionadas con el proceso de búsqueda de información (Kleinberg, 1998; Brin y Page, 1998...). Se centran en la aplicación de un análisis de citas análogo al de publicaciones científicas impresas para descubrir las relaciones que se establecen en la Red y evaluar la calidad de su contenido. Para ello es preciso encontrar una equiparación, respectivamente, entre las citas y referencias en publicación científica y los enlaces que entran (inlinks) y salen (outlinks) de los espacios web. Tal confrontación ha creado controversia entre los investigadores, algunos 
de los cuales sí han aceptado la equiparación (McKiernan, 1996; Larson, 1996...), mientras que otros, sin embargo, tienen un enfoque contrario (Egghe, 2000; Kim, 2000...). En una posición intermedia se sitúa Rousseau (1997), al considerar que, aunque el estudio del análisis de citas en la Web es conceptualmente el mismo que el de la citación tradicional, tiene un sentido ligeramente diferente porque los enlaces y las citas están motivados por cuestiones distintas.

Partiendo de la base de que sí es posible realizar un análisis de citas en la Web (análisis de sitas) y tomando como referencia el conjunto de indicadores basados en enlaces, nuestro objetivo es descubrir el comportamiento de los espacios web de las ANI atendiendo a la cantidad de sitas que se generan entre ellos, centrándonos en las co-sitas e incorporando el PageRank de Google como indicador comparativo.

\subsection{Material y metodología}

El material con el trabajamos está formado por los seis espacios web de las ANI analizados en el apartado 1: tres internacionales — Reuters (http://www.reuters.com), France Press (http://www.afp.com) y Associated Press (http://www.ap.com) $\mathrm{y}$ tres nacionales -Europa Press (http://www.auropapress.es), Colpisa (http://www.colpisa.com) y EFE (http://www.efe.es).

Sobre estos espacios web calculamos dos indicadores de sitas: el análisis de co-sitas y el PageRank de Google (ambos computados en la primera quincena del mes de octubre del 2004). El análisis de co-sitas es una herramienta que ofrece la estructura intelectual del ciberespacio partiendo de la premisa de que cuanto mayor sea la cantidad de veces que dos espacios web aparezcan citados conjuntamente mayor es la probabilidad de que su contenido esté relacionado. En nuestro caso, las co-sitas corresponden al número de veces que aparecen co-sitados por parejas los seis espacios web de las ANI. Para calcularlas usamos las posibilidades de recuperación del motor AltaVista (por ejemplo, link: www.reuters.com AND link: www.afp.com). El siguiente indicador está basado en el algoritmo PageRank del motor Google, que utiliza la estructura de enlaces que ofrece la propia Web para recuperar resultados relevantes de una consulta. El modelo determina la posición individual de cada espacio web en la recuperación teniendo en cuenta no solo el número de espacios web que enlazan con él, sino también el PageRank de los espacios web que lo sitan y el número total de enlaces salientes de estos espacios web sitantes. En este caso, el resultado de este indicador representa el PageRank o puntuación que se muestra en la barra de herramientas de Google para cada uno de los seis espacios web de nuestro estudio.

\subsection{Resultados y discusión}

Con respecto al análisis de co-sitas, lo que obtenemos es una matriz de cositación de 6 x 6, donde las filas y columnas representan a cada uno de los espacios Scire. 11 : 2 (sep.-dic. 2005) 141-152. ISSN 1135-3761. 
web de las ANI (tabla I). Cada celdilla contiene el número de co-sitas entre los dos espacios web que interseccionan en ella. En principio debería ser una matriz simétrica, ya que $\mathrm{A} \cap \mathrm{B}$ y $\mathrm{B} \cap \mathrm{A}$ son dos operaciones lógicamente idénticas, por lo que su resultado debería coincidir (por ejemplo, link: www.colpisa.com AND link: www.reuters.com = link: www.reuters.com AND link: www.colpisa.com). Sin embargo, AltaVista recupera un número ligeramente diferente de páginas. En concreto, se producen variaciones entre los pares siguientes y sus inversos: Colpisa $\cap$ Reuters; EFE $\cap$ France Press; Europa Press $\cap$ France Press; Colpisa $\cap$ Europa Press; EFE $\cap$ Colpisa.

\begin{tabular}{|l|r|r|r|r|r|r|}
\hline \multicolumn{2}{|c}{ Reuters } & \multicolumn{2}{c}{$\begin{array}{c}\text { France } \\
\text { Press }\end{array}$} & \multicolumn{1}{c}{$\begin{array}{c}\text { Associated } \\
\text { Press }\end{array}$} & \multicolumn{2}{c|}{$\begin{array}{c}\text { Europa } \\
\text { Press }\end{array}$} \\
\hline Reuters & & 16.400 & 36.900 & 415 & 39 & 3.080 \\
\hline France Press & 16.400 & & 10.500 & 206 & 40 & 1.900 \\
\hline Associated Press & 36.900 & 10.500 & & 168 & 56 & 1.170 \\
\hline Europa Press & 415 & 194 & 168 & & 204 & 1.990 \\
\hline Colpisa & 41 & 40 & 56 & 216 & & 439 \\
\hline EFE & 3.080 & 1.890 & 1.170 & 1.990 & 449 & \\
\hline
\end{tabular}

Tabla I. Matriz de co-sitación (no simétrica)

Para subsanar este problema, seguimos el mismo método que ya aplicó Smith (1999a) en su investigación sobre el factor de impacto web, es decir, calculamos la media aritmética de los pares diferentes de co-sitas y obtenemos la matriz simétrica que vemos en la tabla II.

\begin{tabular}{|l|r|r|r|r|r|r|}
\hline \multicolumn{2}{c}{ Reuters } & \multicolumn{2}{c}{$\begin{array}{c}\text { France } \\
\text { Press }\end{array}$} & \multicolumn{2}{c}{$\begin{array}{c}\text { Associated } \\
\text { Press }\end{array}$} & \multicolumn{2}{c|}{$\begin{array}{c}\text { Europa } \\
\text { Press }\end{array}$} & \multicolumn{2}{c|}{ Colpisa } & \multicolumn{1}{c|}{ EFE } \\
\hline Reuters & & 16.400 & 36.900 & 415 & 30 & 3.080 \\
\hline France Press & & & 10.500 & 200 & 40 & 1.895 \\
\hline Associated Press & & & & 168 & 56 & 1.170 \\
\hline Europa Press & & & & & 210 & 1.990 \\
\hline Colpisa & & & & & & 444 \\
\hline EFE & & & & & & \\
\hline
\end{tabular}

Tabla II. Matriz de co-sitación (simétrica)

Sobre esta matriz simétrica calculamos, en primer lugar, el número de veces que aparecen co-sitados juntos los espacios web de las ANI internacionales entre 
sí y los de las ANI nacionales, también entre sí (fig. 1). Lo primero que observamos en la figura es que las agencias internacionales aparecen co-sitadas en una proporción mucho mayor que las nacionales, lo que indica que la comunidad web considera mucho más visible a las ANI de carácter internacional. En este caso, es la pareja Reuter-Associated Press la que obtiene un mayor número de co-sitas en la Red, seguida a gran distancia de Reuter-France Press y finalmente de Associated Press-France Press. En el caso nacional, es la pareja EFE-Europa Press la que más veces aparece co-sitada, seguida de EFE-Colpisa y de Europa Press-Colpisa.

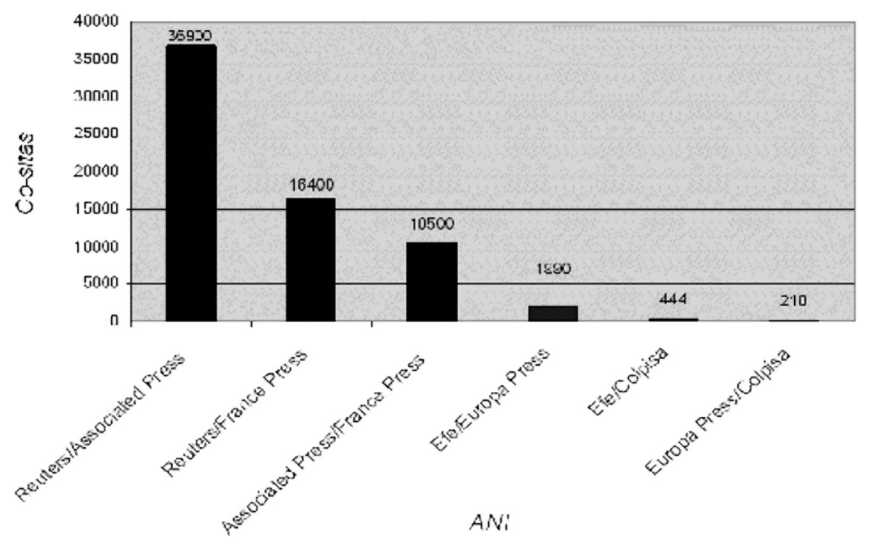

Figura 1. Co-sitación entre las ANI internacionales y las ANI nacionales entre sí

En segundo lugar, comprobamos las veces que las ANI de carácter nacional aparecen co-sitadas con las internacionales (fig. 2). Destaca claramente la pareja

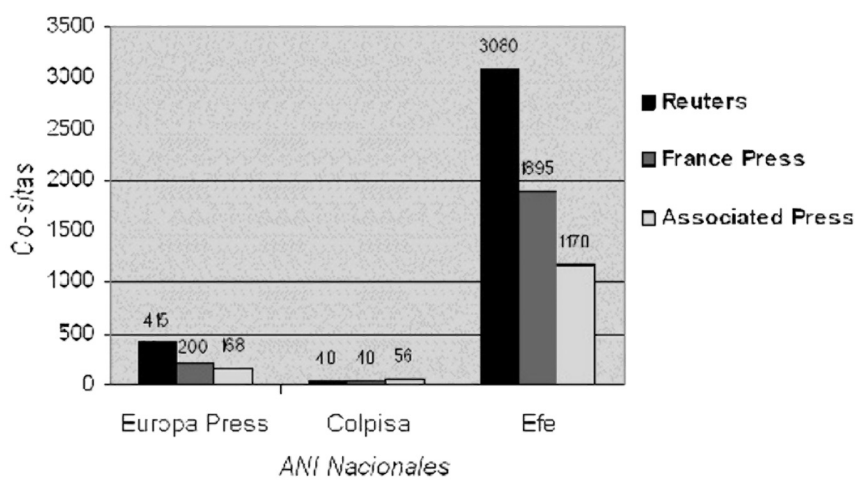

Figura 2. Co-sitación de las ANI nacionales con las internacionales

Scire. $11: 2$ (sep.-dic. 2005) 141-152. ISSN 1135-3761. 
EFE-Reuters con un total de 3080 co-sitas, siendo EFE la agencia española que más veces aparece co-sitada con las agencias internacionales, seguida por Europa Press y en último lugar Colpisa.

La figura 3 muestra la distribución de los espacios web de las ANI teniendo en cuenta el número total de co-sitas de cada web, independiente de las parejas de co-sitación. Como podemos observar, los espacios web de las ANI internacionales son los que más se co-sitan con el resto en una proporción muy elevada. En el caso de las agencias españolas, EFE es la que más se co-sita con el resto, ya sean nacionales o internacionales.

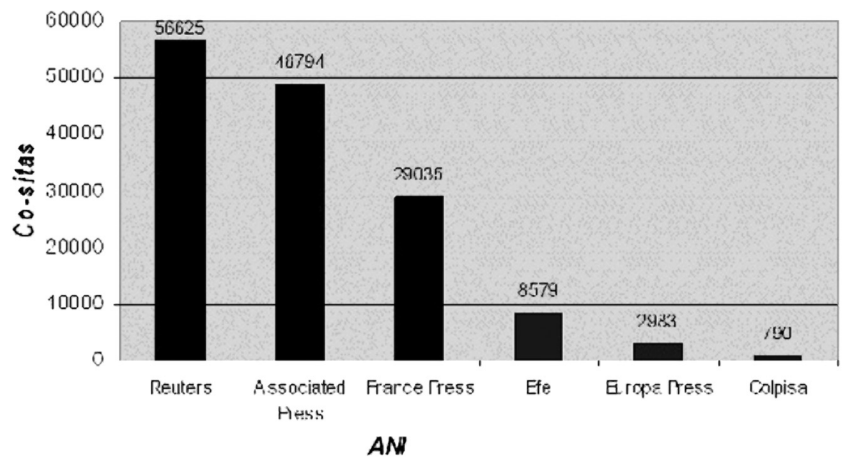

Figura 3. Número total de co-sitas de las ANI

Tras el análisis de co-sitas calculamos el PageRank de Google. En este caso, la distribución que obtenemos sigue el mismo orden que la mostrada en la figura 3 , posicionándose en primer lugar la agencia internacional Reuters con una puntuación de 9 sobre 10 y en última posición Colpisa con un 6 sobre 10, y en posiciones intermedias Associated Press (8), France Press (7), EFE (7) y Europa Press (7). La correlación entre ambas variables es de $0,90$.

\section{Conclusiones}

A continuación se muestran las conclusiones que hemos obtenido en este trabajo.

1. Las co-sitas muestran la relación entre los espacios web que aparecen cositados juntos. Las agencias internacionales Reuters-Associated Press son las que más veces aparecen co-sitadas juntas, lo que indica que existe una estrecha relación entre ellas, motivada, sin duda, por el origen anglosajón de ambas. Asimismo, la pareja de agencias nacionales EFE-Europa Press es la que recibe un mayor número de co-sitas, hecho favorecido por la similitud en el diseño de sus espacios web. 
2. En el caso de las ANI de carácter nacional existe una relación directa entre el número total de co-sitas recibidas y la fecha de aparición de las mismas: EFE (1939, 8579 co-sitas), Europa Press (1957, 2983 co-sitas), Colpisa (1972, 790 co-sitas).

3. Existe una correlación muy elevada entre los dos indicadores basados en sitas que hemos analizado: co-sitas y PageRank (Google).

\section{Referencias}

Aguillo, Isidro F. (1998). Hacia un concepto documental de sede web. // El Profesional de la Información. 7:1-2 (1998) 45-46.

Brin, Sergey; Page, Lawrence (1998). The anatomy of a large-scale hypertextual web search engine. // Computer Networks and ISDN Systems. 30 (1998). URL: <http://www7.scu.edu.au/programme/fullpapers/1921/com1921.htm>. Consultado: 2000-07-30.

Clausen, Helge (1999). Evaluation of library web sites: the Danish case. // The Electronic Library. 17:2 (1999) 83-87.

Codina, Lluis (2000). Evaluación de recursos digitales en línea: conceptos, indicadores y métodos. // Revista Española de Documentación Científica. 23:1 (2000) 9-44.

Egghe, Leo (2000). New informetric aspects of the Internet: some reflections - many problems. // Journal of Information Science. 26:5 (2000) 329-335.

Jiménez-Piano, Marina (2001). Evaluación de sedes web. // Revista Española de Documentación Científica. 24:4 (2001) 405-432.

Kim, Hakoon J. (2000). Motivations for hyperlinking in scholarly electronic articles: a qualitative study. // Journal of the American Society for Information Science. 51:10 (2000) 887-899.

Kleinberg, J. (1998). Authoritative sources in a hyperlinked environment. // Proceedings of the 9th ACM-SIAM Symposium. URL: <http://www.cs.cornell.edu/ home/kleinber/auth.pdf>. Consultado: 2000-10-20.

Larson, Ray R. (1996). Bibliometrics of the World Wide Web: and exploratory analysis of the intellectual structure of cyberspace. // Proceedings of the 59th Annual Meeting of the American Society for Information Science. URL: <http://sherlock.berkeley.edu/ asis96/asis96.html>. Consultado: 1999-02-24.

Manfredi-Mayoral, Juan L. (2000). Manual de producción periodística. Sevilla: MAD, 2000.

McKiernan, Gerry (1996). CitedSites(sm): Citation Indexing of Web Resources. URL: $<$ http://www.public.iastate.edu/ CYBERSTACKS/Cited.htm>. Consultado: 200002-02.

Novljan, Silva; Zumer, Maja (2004). Web pages of Slovenian public libraries: evaluation and guidelines. // Journal of Documentation. 60:1 (2004) 62-76.

Rousseau, Ronald (1997). Sitations: an exploratory study. // Cybermetrics: International Journal of Scientometrics, Informetrics and Bibliometrics. URL: <http://www.cindoc.csic.es/ cybermetrics/articles/v1i1p1.html>. Consultado: 1999-02-24.

Scire. $11: 2$ (sep.-dic. 2005) 141-152. ISSN 1135-3761. 


\section{2}

Smith, Alastair G. (1997). Testing the Surf: criteria for evaluating Internet information resources. // The Public-Access Computer Systems Review. URL: <http://info.lib.uh.edu/ pr/v8/n3/smit8n3.html>. Consultado: 2002-02-28.

Smith, Alastair G. (1999a). A tale of two web spaces: comparing sites using web impact factors. // Journal of Documentation. 55:5 (1999) 577-592.

Smith, Alastair G. (1999b). The impact of web sites: a comparison between Australasia and Latin America (1999). URL: <http://www.vuw.ac.nz/ agsmith/publns/austlat>. Consultado: 2001-05-14.

Thelwall, Mike (2001). Exploring the link structure of the Web with network diagrams. // Journal of Information Science. 27:6 (2001) 393-401.

Thelwall, Mike; Smith, Alastair G. (2002). Interlinking between Asia-Pacific University web sites. // Scientometrics. 55:3 (2002) 363-376. 